\title{
An Hour Ahead Electricity Price Forecasting with Least Square Support Vector Machine and Bacterial Foraging Optimization Algorithm
}

\author{
Intan Azmira Wan Abdul Razak', Izham Zainal Abidin², Yap Keem Siah, \\ Aidil Azwin Zainul Abidin ${ }^{4}$, Titik Khawa Abdul Rahman', Nurliyana Baharin ${ }^{6}$, Mohd. Hafiz Bin Jali ${ }^{7}$ \\ ${ }^{1,6,7}$ Faculty of Electrical Engineering, Universiti Teknikal Malaysia Melaka, Malacca, Malaysia \\ 2,3,4 College of Engineering, National Energy University, Selangor, Malaysia \\ ${ }^{3}$ Faculty of Engineering Girl Campus, King Abdulaziz University, Jeddah, Saudi Arabia
}

\begin{tabular}{l} 
Article Info \\
\hline Article history: \\
Received Nov 7, 2017 \\
Revised Jan 20, 2018 \\
Accepted Feb 20, 2018 \\
\hline Keywords: \\
Bacterial \\
Electricity Price Forecasting \\
Foraging Optimization \\
Hour Ahead Forecast \\
MAPE \\
Support Vector Machine
\end{tabular}

Support Vector Machine

\begin{abstract}
Predicting electricity price has now become an important task in power system operation and planning. An hour-ahead forecast provides market participants with the pre-dispatch prices for the next hour. It is beneficial for an active bidding strategy where amount of bids can be reviewed or modified before delivery hours. However, only a few studies have been conducted in the field of hour-ahead forecasting. This is due to most power markets apply two-settlement market structure (day-ahead and real time) or standard market design rather than single-settlement system (real time). Therefore, a hybrid multi-optimization of Least Square Support Vector Machine (LSSVM) and Bacterial Foraging Optimization Algorithm (BFOA) was designed in this study to produce accurate electricity price forecasts with optimized LSSVM parameters and input features. So far, no works has been established on multistage feature and parameter optimization using LSSVM-BFOA for hour-ahead price forecast. The model was examined on the Ontario power market. A huge number of features were selected by five stages of optimization to avoid from missing any important features. The developed LSSVM-BFOA shows higher forecast accuracy with lower complexity than most of the existing models.
\end{abstract}

Copyright $@ 2018$ Institute of Advanced Engineering and Science. All rights reserved.

\section{Corresponding Author:}

Intan Azmira Wan Abdul Razak, Faculty of Electrical Engineering, Universiti Teknikal Malaysia Melaka, Hang Tuah Jaya, 76100 Durian Tunggal, Melaka, Malaysia.

Email: intan.azmira@utem.edu.my

\section{INTRODUCTION}

Hour-ahead electricity price prediction is crucial to market participants in deregulated electricity market to produce an appropriate bidding plan where the quantity of bids can be revised or changed prior to the dispatch hour. However, only a few studies have been conducted in the field of hour-ahead price forecasting. This is because most power markets run two-settlement market structure (day-ahead and real time) or standard market design rather than single-settlement system (real time).

Previous researcers proposed various methods such as time series model of Multivariate Adaptive Regression Splines (MARS) [1], Levenberg-marquardt (LM) back propagation [2], and Input-Output Hidden Markov Model (IOHMM) [3]. Meanwhile, a hybrid method are also developed such as recurrent neural networks (RNN) and excitable dynamics [4] and a hybrid model of Autoregressive Moving Average Exogenous (ARMAX), adaptive wavelet neural network (AWNN), and Generalized Autoregressive Conditional Heteroskedasticity (GARCH) to treat linear and nonlinear structures of price series [5]. Other 
Neural Network (NN) techniques were also modelled such as Expectation Maximization (EM) technique for maximum likelihood estimation of RNN (RNN-EM) [6], Multi-layer Perceptron NN trained by Extended Kalman Filter (MLP-EKF) and EM (MLP-EM) [7] and Extended Kalman Filter for RNN (RNN-EKF) [8]. A Generalized Regression Neural Network (GRNN) was developed by [9] and Discrete Cosine Transforms Input Featured Feed-Forward Neural Network (DCT-FFNN) model was proposed by [10]. The same researchers further improved the forecast by creating classification models using three layered FFNN, Cascade-Forward Neural Network (CFNN) trained by the LM algorithm, and GRNN models [11].

Most existing techniques have good predictions during normal circumstances or without a surge event; but when spikes are present, forecast predictions become large. Hence, this study introduces a new technique in electricity price forecast by developing hour-ahead electricity price forecasting model with Least Square Support Vector Machine (LSSVM) and Bacterial Foraging Optimization Algorithm (BFOA). BFOA has a fast convergence [12] and has been explored in many fields such as face recognition [13], [14], biometric authentication [15], multimodal function [16], [17], and flexible manufacturing systems (FMS) [18]. Furthermore, researchers in control and power system developed BFOA models for Static Synchronous Series Compensator (SSSC) Damping Controller Design [19], robotic manipulator workspace optimization [20], three phase induction motor and electricity load forecasting [28], [34]. To the best of the authors' review, no literature has been found on the combination of LSSVM and BFOA in the electricity price forecast. Furthermore, the approach of multistage feature and parameter selections using a single optimization method has not been investigated yet. With a single optimization method of BFOA, the input features and LSSVM parameters are simultaneously optimized through five-stage optimization approach. This method is shown to provide better prediction accuracy compared to most existing models, which can contribute for decision-making and hourly market operation.

\section{TOPOLOGY OF SVM, LSSVM AND BFOA}

This section provides topologies of SVM, LSSVM and BFOA which were applied in this study.

\subsection{SVM and LSSVM}

SVM can reduce over-fitting, local minima problems [26], and able to deal with high dimensional input spaces splendidly. However, the main drawback of SVM is the high computational complexity due to constrained optimization programming. Hence, Least Squares Support Vector Machine (LSSVM) has been proposed to reduce the SVM computational burden, which applies with equality rather than the inequality constraints. LSSVM solves a system of linear equations to cater Quadratic Programming (QP) issues that increase computational speed [27], [28]. The linear system, namely as Karush- Kuhn-Tucker (KKT), is simpler than QP system. LSSVM also keeps the SVM principle, which has good generalization capability. LSSVM reduces the Sum Square Errors (SSEs) of training data sets and concurrently diminishing margin error. The LSSVM model for regression is represented as in (1):

$$
f(x)=\sum_{k=1}^{N} \alpha_{k} K\left(x, x_{k}\right)+b
$$

\subsection{BFOA}

The E.coli bacteria, which is present in human's intestines has unique foraging activities during locating and ingesting nutrient or food. BFOA imitates this mechanism through four main steps during foraging; namely, chemotaxis, swarming, reproduction, and elimination-dispersal. The flow of BFOA applied in this work is shown in Figure 1.

In the chemotaxis step, bacteria look for nutrients to maximize the energy intake while foraging by taking small steps (chemotaxis) and interacting with other bacteria by sending attractant signal to form flocks; or repellent signal to move individually. They tumble or swim to search nutrient but keep away from unsafe places. Therefore, suppose that $\theta^{i}(j, k, l)$ is the $i$-th bacterium position at $j$-th chemotactic, $k$-th reproduction, and $l$-th elimination-dispersal step, the position of each bacterium after swimming or tumbling can be defined as (2):

$$
\theta^{i}(j+1, k, l)=\theta^{i}(j, k, l)+C(i) \frac{\Delta(i)}{\sqrt{\Delta^{T}(i) \Delta(i)}}
$$




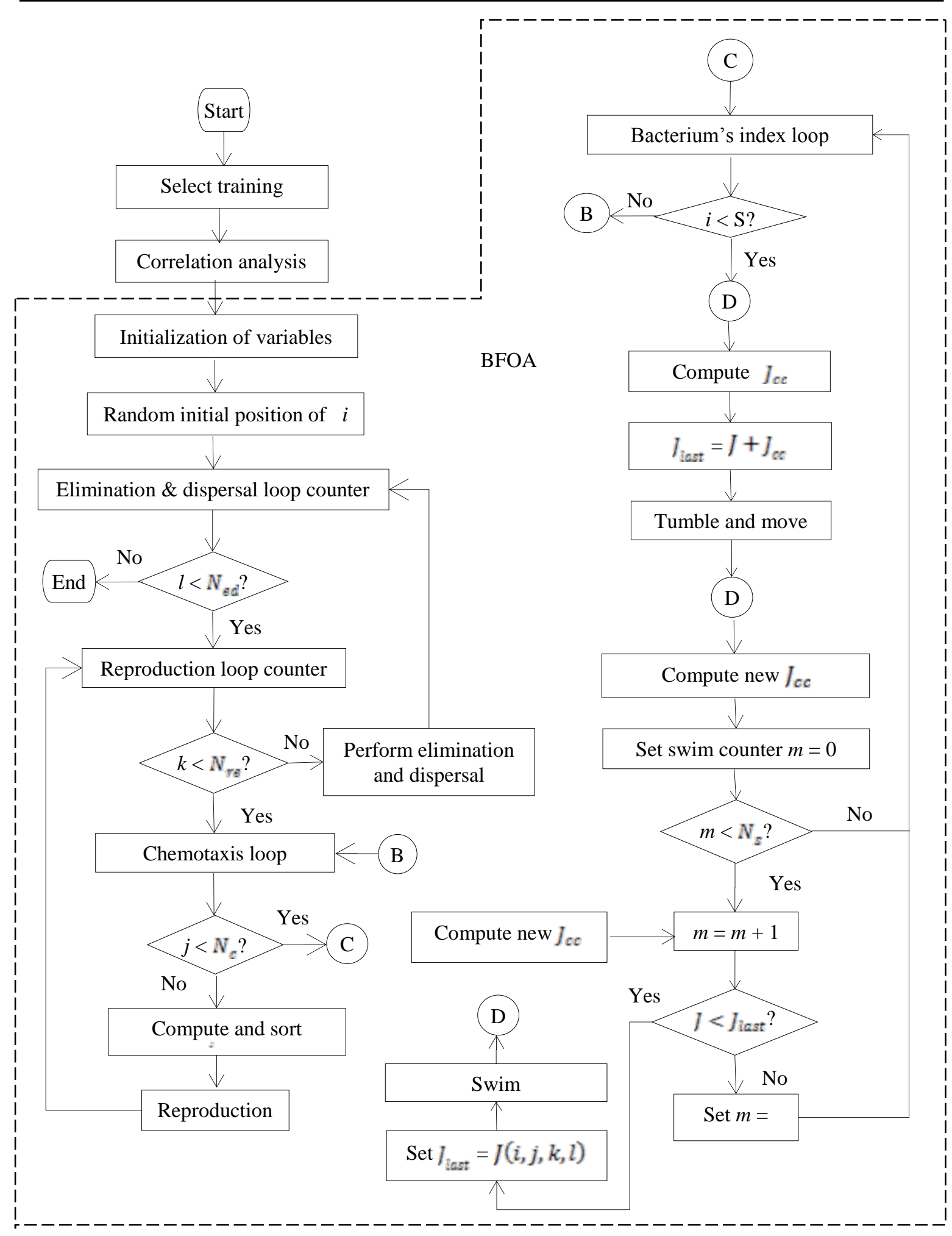




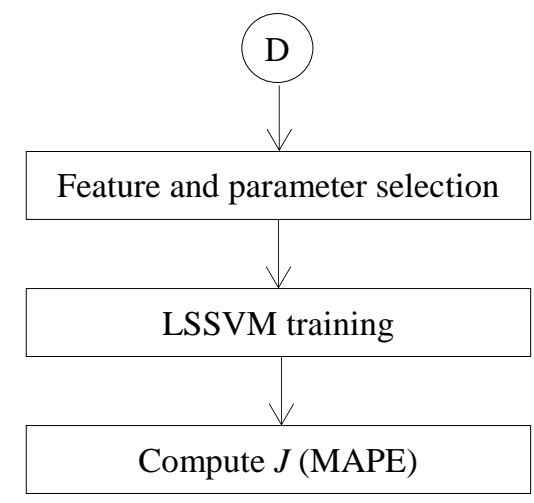

Figure 1: Flowchart of LSSVM-BFOA Model Development

Where $C(\mathrm{i})$ is the measure of the step taken during tumbling or swimming, and $\Delta$ is the vector in a random direction where the elements lie in position $[-1,1]$. The objective function or actual cost for every location of bacterium $i$ is calculated and represented as $J(i, j, k, l)$. During swarming step, a bacterium that has found a good nutrient source during its search may attract other bacteria to form flocks. Instead, the repellent signal may be released to ensure that the bacteria are not too close to each other. The cell-to-cell attraction and repellent of E.Coli swarm can be represented as $J_{\mathrm{cc}}$, the objective function value to be added to the current objective function which will decrease the final objective function. When food is sufficient and the temperature is appropriate, the healthiest or good bacteria will grow in length and break in the middle to form the self-replicating which contributes to the next generation while the least healthy bacteria die. This activity is known as reproduction. Thus, BFOA uses this phenomenon by structuring the best objective function in the ascending order and maintaining half of the population size to reproduce while the other half is eliminated. The last step is the elimination-dispersal where the chemotactic process can be dissolved and the bacteria spread to new positions when a sudden change in the environment exists.

\section{RESEARCH METHOD}

In Ontario, the electricity market is operated by the Independent Electricity Systems Operator (IESO), which controls the operation of power systems, predicts short-term demand and electricity supply, and manages real time market electricity prices. Due to the single settlement real-time power market, Ontario is reported to be one of the most volatile markets in the world and hence it is a big challenge for electric price forecasters. [29]. Appropriate selection of features affects the efficiency and accuracy of predictions. The input features used in this study are as in [30], where correlation analysis is performed to observe the significant features for forecasting. The total features are [(15 days x 24 hours price $)+(15$ days $\times 24$ hours demand $)+1$-hour pre-dispatch price $=721$ ]. Noted that this method is an initial process to filter or reduce the number of features to be optimized by BFOA. Hybrid model of LSSVM-BFOA was developed with fivestage optimization of feature and parameter. The flow of BFOA applied in this work is shown in Figure 1.

During the first stage, all 721 features are applied and the BFOA selects certain number of significant features to be fed into the LSSVM. At the same time, BFOA optimizes the LSSVM parameters; gamma $(\gamma)$ and sigma $(\sigma)$. During the second stage of optimization, BFOA optimizes the features and parameters that have been selected from the first stage of optimization. These steps are repeated for the next stage of optimization until no improvement has been observed in the fitness value or Mean Absolute Percentage Error (MAPE). MAPE and Mean Absolute Error (MAE) are expressed as in (3) and (4); respectively, where $P_{\text {actual }}$ and $P_{\text {forecast }}$ are the actual and forecasted HOEP at hour $t$, respectively, while $N$ is the number of hours.

$$
\begin{gathered}
\text { MAPE }=\frac{100}{N} \times \sum_{t=1}^{N} \frac{\left|P_{\text {actualt }}-P_{\text {forecast }}\right|}{P_{\text {actualt }}} \\
\text { MAE }=\frac{1}{N} \times \sum_{t=1}^{N}\left|P_{\text {actualt }}-P_{\text {forecast } t}\right|
\end{gathered}
$$




\section{RESULTS AND ANALYSIS}

In comparison with previous researchers, six predictive models were developed to represent throughout 2004. Each model is trained with ten weeks of training samples prior to the forecasting week as presented in [30]. Table 1 presents the result for all test weeks and optimization stages. It can be noted that the average MAPE decreases after each level of optimization. The best MAPEs are obtained during the fifth stage of optimization. Table 2 reveals the network configurations for all test weeks during the fifth stage of optimization. The BFOA parameters must be chosen properly by trial and error method [31], [32], [33].

The main optimization process occurs during chemotaxis activity where the objective function is calculated for each bacterium. Too small value of $N_{\mathrm{c}}$ may trap the bacteria into local minima. $N_{\mathrm{s}}$ value must be smaller than $N_{\mathrm{c}}$ value. Although the swimming activity occurs in chemotaxis loop, the swimming counter will be terminated if the MAPE produced is greater than the previous MAPE. The value of $p_{\text {ed }}$ is set as 0.25 since too large value can increase computational burden due to an extensive search. Meanwhile, the $N_{\text {re }}$ should not be too small as it may cause premature convergence. As in general, increasing the size of $S, N_{\text {ed }}$, $N_{\text {re }}$, and $N_{\mathrm{c}}$ may increase the computational burden, but hopefully it may improve the optimization process since bacteria have a wider search space. Furthermore, the developed models of LSSVM-BFOA are compared with other existing models as tabulated in Table 3. Based on the observation of Table 3, the LSSVM-BFOA model shows better result than other available models except for LSSVM+GA [30] and RNN with excitable dynamics [4] models. However, the LSSVM-BFOA model is considered as comparable since the differences of MAPE are only $0.55 \%$ [4] and 3.6\% [30]. In comparison, the RNN model has a more complex structure, which is designed to handle spiky and non-spiky price regions.

Table 1. MAPE of LSSVM-BFOA for Hour-Ahead Forecast

\begin{tabular}{|c|c|c|c|c|c|c|c|}
\hline \multirow{2}{*}{ Optimization Stage } & \multicolumn{7}{|c|}{ MAPE (\%) } \\
\hline & Week 1 & Week 2 & Week 3 & Week 4 & Week 5 & Week 6 & Average \\
\hline Stage 1 & 16.58 & 15.30 & 12.51 & 26.89 & 17.36 & 21.67 & 18.39 \\
\hline Stage 2 & 13.53 & 13.01 & 10.69 & 19.92 & 15.83 & 14.48 & 14.58 \\
\hline Stage 3 & 11.21 & 10.82 & 7.99 & 13.94 & 14.91 & 11.90 & 11.80 \\
\hline Stage 4 & 13.53 & 10.15 & 7.10 & 11.63 & 13.43 & 10.76 & 11.11 \\
\hline Stage 5 & 11.87 & 10.31 & 8.55 & 11.21 & 13.30 & 10.78 & 11.00 \\
\hline Stage 6 & 11.79 & 10.34 & 8.40 & 11.68 & 13.25 & 11.20 & 11.11 \\
\hline
\end{tabular}

Table 2. Selected configuration of LSSVM-BFOA for Hour-Ahead Forecast

\begin{tabular}{|c|c|c|c|c|c|c|}
\hline & \multicolumn{6}{|c|}{ Test data } \\
\hline & Week 1 & Week 2 & Week 3 & Week 4 & Week 5 & Week 6 \\
\hline$S$ & 20 & 20 & 20 & 20 & 16 & 20 \\
\hline$N_{\mathrm{c}}$ & 50 & 100 & 50 & 50 & 50 & 50 \\
\hline$N_{\mathrm{s}}$ & \multicolumn{6}{|c|}{5} \\
\hline$N_{\text {re }}$ & \multicolumn{6}{|c|}{4} \\
\hline$N_{\text {ed }}$ & \multicolumn{6}{|c|}{2} \\
\hline$p_{\text {ed }}$ & \multicolumn{6}{|c|}{0.25} \\
\hline Gamma & 5.21 & 15.72 & 16.22 & 18.53 & 0.85 & 1.54 \\
\hline Sigma & 0.99 & 2.24 & 7.62 & 10.30 & 1.07 & 2.98 \\
\hline Selected Features & 17 & 22 & 22 & 16 & 22 & 22 \\
\hline Regression (R) & 0.81 & 0.89 & 0.88 & 0.93 & 0.80 & 0.85 \\
\hline MAE & 5.54 & 5.01 & 4.48 & 5.00 & 9.37 & 7.18 \\
\hline
\end{tabular}

Table 3. MAPE for Hour-Ahead Forecast in the Ontario Electricity Market

\begin{tabular}{|c|c|c|c|c|c|c|c|c|c|}
\hline \multirow{2}{*}{ Ref. } & \multirow{2}{*}{ Year } & \multirow{2}{*}{ Method } & \multicolumn{6}{|c|}{ Test week } & \multirow{2}{*}{$\begin{array}{l}\text { Average } \\
\text { MAPE }\end{array}$} \\
\hline & & & 1 & 2 & 3 & 4 & 5 & 6 & \\
\hline \multirow{3}{*}{$\begin{array}{c}{[30]} \\
{[4]}\end{array}$} & \multirow{3}{*}{$\begin{array}{l}2016 \\
2013\end{array}$} & LSSVM-BFOA & 11.87 & 10.31 & 8.55 & 11.21 & 13.30 & 10.78 & 11.00 \\
\hline & & LSSVM-GA & 7.55 & 7.45 & 5.55 & 7.88 & 7.21 & 8.77 & 7.40 \\
\hline & & $\begin{array}{l}\text { RNN - Expectation Maximization } \\
\text { algorithm (RNN-EM) }\end{array}$ & 15.09 & 15.16 & 10.52 & 10.21 & 15.78 & 15.71 & 13.72 \\
\hline \multirow[t]{3}{*}{ [6] } & \multirow[t]{2}{*}{2011} & $\begin{array}{l}\text { RNN - Extended Kalman Filter } \\
\text { (RNN-EKF) }\end{array}$ & 16.01 & 16.54 & 11.89 & 11.96 & 16.59 & 16.45 & 14.91 \\
\hline & & MLP-EKF & 16.83 & 16.74 & 12.64 & 15.25 & 16.77 & 16.96 & 15.87 \\
\hline & \multirow{4}{*}{2006} & MLP-EM & 15.48 & 15.39 & 11.87 & 12.07 & 16.78 & 16.73 & 14.72 \\
\hline \multirow{3}{*}[1]{} & & MARS (case 1) & 13.3 & 12.9 & 9.4 & 14.4 & 12.9 & 15.5 & 13.07 \\
\hline & & MARS (case 2) & 12.5 & 12.3 & 8.6 & 11.7 & 11.8 & 13.9 & 11.80 \\
\hline & & IESO & 23.78 & 25.26 & 10.41 & 16.22 & 22.06 & 23.51 & 20.21 \\
\hline
\end{tabular}




\section{CONCLUSION}

In the field of electricity price forecasting, accuracy of the prediction is the main issue. Nevertheless, predictive models with high precision usually need a more complex model structure. Apart from that, selection of features and parameter are also important tasks during the forecast model development. Hence, a hybrid model of LSSVM-BFOA for hour-ahead electricity price forecast was developed in this study. BFOA completes both feature and LSSVM parameter optimizations simultaneously. Through several levels of optimization, the amount of inputs to be fed into the LSSVM structure will be reduced and at the same time the value of the LSSVM parameter is refined. Although the accuracy of the LSSVM-BFOA is slightly lower than the previous best models, the developed model shows simpler structure and provides better MAPE than most of the existing models in the Ontario power market. In addition, until recently, no study has investigated the application of BFOA in electricity price forecasting. This contribution can help market members to bid effectively, maintain efficient daily operations, and ultimately increase the company's profits. Therefore, some refinement and modification on the LSSVM-BFOA model could be performed in future to reduce the forecast error. The forecast accuracy may be enhanced by proper selection of their parameters such as the number of bacteria $(S)$, number of chemotactic steps $\left(N_{\mathrm{c}}\right)$, number of steps taken during swimming $\left(N_{\mathrm{s}}\right)$, number of reproduction steps $\left(N_{\mathrm{re}}\right)$, number of elimination-dispersal steps $\left(N_{\mathrm{ed}}\right)$, probability of elimination-dispersal $\left(p_{\text {ed }}\right)$, attractant depth $\left(d_{\text {attract }}\right)$, and attractant width $\left(w_{\text {attract }}\right)$.

\section{ACKNOWLEDGEMENTS}

This study is supported in part by the Short Term Grant (PJP) provided by Universiti Teknikal Malaysia, Melaka (PJP/2015/FKE(2A)/S01400). We would like to dedicate our appreciation to Universiti Teknikal Malaysia, Melaka (UTeM) for providing financial and moral support throughout conducting this study

\section{REFERENCES}

[1] H. Zareipour, K. Bhattacharya, and C. a. Canizares, "Forecasting the hourly Ontario energy price by multivariate adaptive regression splines," in 2006 IEEE Power Engineering Society General Meeting, 2006, pp. 1-7.

[2] K. B. Sahay, "One hour ahead price forecast of Ontario electricity market by using ANN," in 2015 International Conference on Energy Economics and Environment (ICEEE), 2015, pp. 1-6.

[3] A. Mateo, A. Muñoz, and J. García-González, "Modeling and Forecasting Electricity Prices with Input/Output Hidden Markov Models," IEEE Trans. Power Syst., vol. 20, no. 1, pp. 13-24, 2005.

[4] V. Sharma and D. Srinivasan, "A hybrid intelligent model based on recurrent neural networks and excitable dynamics for price prediction in deregulated electricity market," Eng. Appl. Artif. Intell., vol. 26, no. 5-6, pp. $1562-1574,2013$.

[5] L. Wu and M. Shahidehpour, "A Hybrid Model for Day-Ahead Price Forecasting," IEEE Trans. Power Syst, vol. 25, no. 3, pp. 1519-1530, 2010.

[6] D. Mirikitani and N. Nikolaev, "Nonlinear maximum likelihood estimation of electricity spot prices using recurrent neural networks," Neural Comput. Appl., vol. 20, no. 1, pp. 79-89, Feb. 2011.

[7] J. F. G. de Freitas, M. Niranjan, and A. H. Gee, "Dynamic learning with the EM algorithm for neural networks," $J$. VLSI Signal Process., vol. 26, no. 1/2, pp. 119-131, 2000.

[8] C. M and B. L, "Simple recurrent network trained by RTRL and extended Kalman filter algorithms," Neural Netw. World, vol. 13, no. 3, pp. 223-234, 2003.

[9] S. Anbazhagan, "Day-Ahead Price Forecasting in Asia $\hat{a} €^{T M}$ S First Liberalized Electricity Market Using Artificial Neural Networks," in Second International Conference on Sustainable Energy and Intelligent System (SEISCON 2011), 2011, vol. 4, no. 4, pp. 476-485.

[10] S. Anbazhagan and N. Kumarappan, "Day-ahead deregulated electricity market price forecasting using neural network input featured by DCT," Energy Convers. Manag., vol. 78, pp. 711-719, 2014.

[11] S. Anbazhagan and N. Kumarappan, "A neural network approach to day-ahead deregulated electricity market prices classification,” Electr. Power Syst. Res., vol. 86, pp. 140-150, 2012.

[12] V. P. Sakthivel, R. Bhuvaneswari, and S. Subramanian, "Design optimization of three-phase energy efficient induction motor using adaptive bacterial foraging algorithm," COMPEL Int. J. Comput. Math. Electr. Electron. Eng., vol. 29, no. 3, pp. 699-726, 2010.

[13] R. Jakhar, N. Kaur, and R. Singh, "Face Recognition Using Bacteria Foraging Optimization-Based Selected Features," Int. J. Adv. Comput. Sci. Appl., vol. 1, no. 3, pp. 106-111, 2011

[14] S. Arivalagan and K. Venkatachalapathy, "Face Recognition based on a Hybrid Meta-heuristic Feature Selection Algorithm,” Int. J. Comput. Appl., vol. 55, no. 17, pp. 18-22, 2012.

[15] M. Karnan and N. Krishnaraj, "A Model to Secure Mobile Devices Using Keystroke Dynamics through Soft Computing Techniques," Int. J. Soft Comput. Eng., vol. 2, no. 3, pp. 71-75, 2012.

[16] K. M. Bakwad, S. S. Pattnaik, B. S. Sohi, S. Devi, B. K. Panigrahi, and S. V. R. S. Gollapudi, "Multimodal Function Optimization Using Synchronous Bacterial Foraging Optimization Technique," IETE J. Res., vol. 56, pp. $80-87,2010$ 
[17] A. Kasaiezadeh, A. Khajepour, and S. L. Waslander, "Spiral Bacterial Foraging Optimization method," in Proceedings of the 2010 American Control Conference, 2010, pp. 4845-4850.

[18] B. D. and B. D. S. A.V.S.Sreedhar Kumar, V.Veeranna, "A MATLAB GUI Tool for Optimization of FMS Scheduling using Conventional and Evolutionary Approaches," Int. J. Curr. Eng. Technol., vol. 3, no. 5, pp. 17391744, 2013.

[19] E. S. Ali and S. M. Abd-Elazim, "Hybrid BFOA-PSO approach for SSSC damping controller design," 2013 International Conference on Control, Decision and Information Technologies (CoDIT). Ieee, pp. 464-469, May2013.

[20] S. Panda, D. Mishra, B. B. Biswal, and M. Tripathy, "Revolute manipulator workspace optimization using a modified bacteria foraging algorithm: A comparative study," Eng. Optim., vol. 46, pp. 181-199, 2013.

[21] M. Ulagammai, P. Venkatesh, P. S. Kannan, and N. Prasad Padhy, "Application of bacterial foraging technique trained artificial and wavelet neural networks in load forecasting," Neurocomputing, vol. 70, pp. 2659-2667, 2007.

[22] Y. Li, "Short-Term Load Forecasting Based on LS-SVM Optimized by BCC Algorithm," in 2009 15th International Conference on Intelligent System Applications to Power Systems, 2009, no. 2, pp. 1-5.

[23] Z. B. Shi, Y. Li, and T. Yu, "Short-term load forecasting based on LS-SVM optimized by bacterial colony chemotaxis algorithm," in 2009 International Conference on Information and Multimedia Technology, ICIMT 2009, 2009, no. 3, pp. 306-309.

[24] V. Hoo and J. Han, "South China Load Forecasting based on BFO," Adv. Inf. Technol. and Management, vol. 1, no. 1, pp. 33-37, 2012.

[25] Y. Zhang, L. Wu, and S. Wang, "Bacterial Foraging Optimization Based Neural Network for Short-term Load Forecasting," J. Comput. Inf. Syst., vol. 7, pp. 2099-2105, 2010.

[26] G. Xie, S. Wang, Y. Zhao, and K. K. Lai, "Hybrid approaches based on LSSVR model for container throughput forecasting: A comparative study,” Appl. Soft Comput., vol. 13, no. 5, pp. 2232-2241, May 2013.

[27] H. Wang and D. Hu, "Comparison of SVM and LS-SVM for Regression," in 2005 International Conference on Neural Networks and Brain, 2005, no. 5, pp. 279-283.

[28] S. Li and L. Dai, "Classification of gasoline brand and origin by Raman spectroscopy and a novel R-weighted LSSVM algorithm," Fuel, vol. 96, pp. 146-152, Jun. 2012.

[29] H. Zareipour, K. Bhattacharya, and C. a. Cañizares, "Electricity market price volatility: The case of Ontario," Energy Policy, vol. 35, no. 9, pp. 4739-4748, Sep. 2007.

[30] I. A. W. A. Razak, I. Z. Abidin, K. S. Yap, A. A. Z. Abidin, T. K. A. Rahman, and M. N. M. Nasir, "A novel hybrid method of LSSVM-GA with multiple stage optimization for electricity price forecasting," in 2016 IEEE International Conference on Power and Energy (PECon), 2016, pp. 390-395.

[31] K. Prabaakaran, S. Jaisiva, A. Selvakumar, and K. S. Kumar, "Implementation of Bacterial Foraging Algorithm for Enhancing Voltage Profile and Loss Minimization using Static Var Compensator," Int. J. Res. Dev. Eng., vol. 1, no. 3, pp. 43-50, 2013.

[32] P. K. Majhi, R. ; Bhubaneswar ; Panda, G.; Sahoo, G. ; Dash, "Stock market prediction of S\&P 500 and DJIA using Bacterial Foraging Optimization Technique," in IEEE Congress on Evolutionary Computation, 2007, pp. 2569 2575.

[33] J. Dang, A. Brabazon, M. O. Neill, and D. Edelman, "Option Model Calibration Using a Bacterial Foraging Optimization Algorithm," in Applications of Evolutionary Computing, 2008, pp. 113-122.

\section{BIOGRAPHIES OF AUTHORS}
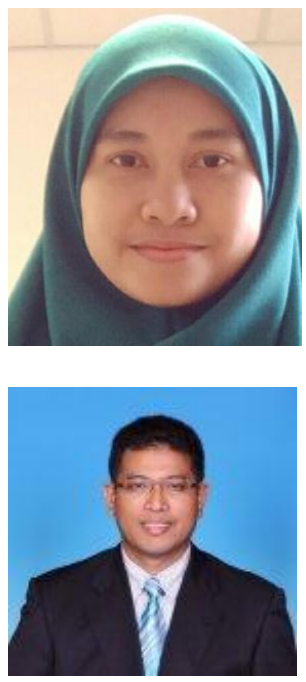

Intan Azmira Wan Abdul Razak received the B.Sc. degree in Electrical Engineering from Universiti Teknikal Malaysia Melaka (UTeM), Malaysia in 2006, completed her Master engineering studies in Electrical - Power at Universiti Teknologi Malaysia (UTM) in 2008 and $\mathrm{PhD}$ from The National Energy University (UNITEN), Malaysia in 2017. Currently she works as senior lecturer at Industrial Power Department at the Faculty of Electrical Engineering at UTeM. Her main interest is power system planning and optimization.

Izham Zainal Abidin received the Ordinary National Diploma in Engineering from Coventry Technical College in 1994, B.Eng in Electrical Engineering from University of Southampton in 1997, and PhD in Electrical Engineering (Power) from Strathclyde University in 2002. Currently he is professor at College of Engineering at UNITEN. His main interest is power system analysis and stability. 


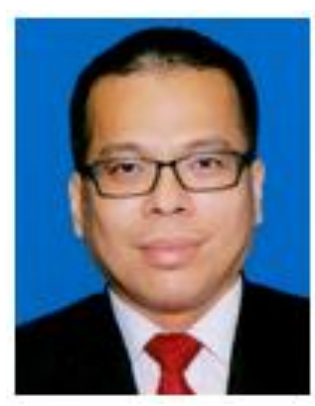

Yap Keem Siah received B.Eng in Electrical Engineering (Hons) from Universiti Teknologi Malaysia (UTM) in 1998, Master of Engineering (Electrical) from Universiti Teknologi Malaysia (UTM) in 2000, and PhD from Universiti Sains Malaysia (USM) in 2010. Currently he is professor at College of Engineering at UNITEN. His main interests are computational intelligence and signal processing.

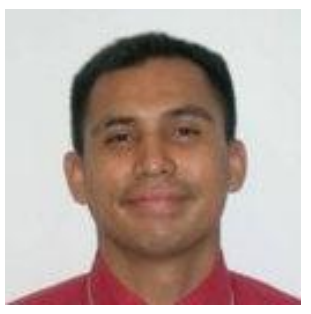

Aidil Azwin Zainul Abidin received Bachelor of Science in Electrical Engineering from Indiana University Perdue University Indianapolis in 1999, Masters in Electrical Engineering from Universiti Tenaga Nasional in 2005, and PhD in Engineering from UNITEN in 2012. Currently he is senior lecturer at College of Engineering at UNITEN. His main interests are renewable energy and image processing.

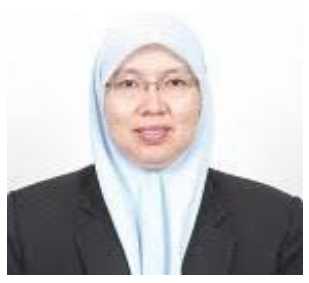

Titik Khawa Abdul Rahman is a Professor in Electrical Engineering. Her main interests are Power System Operation and Artificial Intelligence Application.

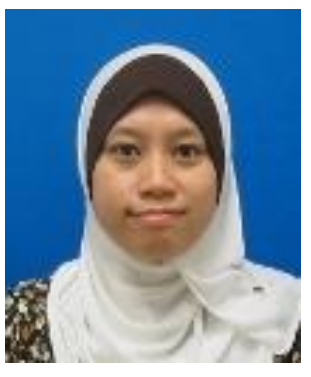

Nurliyana Baharin received B.Eng. (Electrical) from Universiti Teknologi Malaysia (UTM) and M. Eng. in Electrical Engineering, UNITEN. Currently she is lecturer at UTeM. Her main interests are power system analysis and power quality.

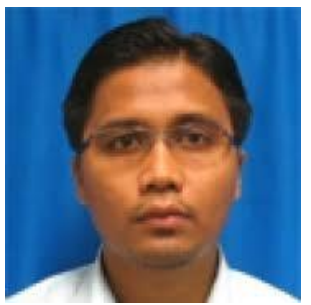

Mohd Hafiz Jali received B.Sc. degree in electrical and electronic engineering with first class honours from MARA University of Technology (UiTM), Malaysia in 2007 and completed his Master engineering studies with distinction in Industrial Electronics and Control at University of Malaya (UM), Malaysia. He was with the automotive company, Proton Sdn. Bhd from 2007 to 2010 as a Test Engineer under the Complete Vehicle Testing (CVT) department. In 2012, he was appointed as a Lecturer at Universiti Teknikal Malaysia Melaka (UTeM), Malaysia. He is currently a PhD student under Nanotechnology and Sensor Laboratory at Faculty of Engineering, University Malaya. 